\title{
Transferência de colônias de vespas sociais (Hymenoptera, Vespidae) em área urbana ${ }^{1}$
}

\author{
Transfer of social wasps colonies (Hymenoptera, Vespidae) in urban areas
}

\author{
Mateus Fajardo de Freitas Salviato Detoni ${ }^{2}$ \\ Angie Zuleidi Amezquita Mayorquin ${ }^{3}$ \\ Fabio Prezoto ${ }^{4}$
}

DOI: https://doi.org/10.34019/2179-3700.2018.v18.29866

\begin{abstract}
Resumo
As vespas sociais são pouco utilizadas como controladores biológicos, em parte por desconhecimento e má fama, e também por que não se sabe como as espécies reagem à transferência de seus ninhos. Assim, nosso estudo visou a transferência de colônias da vespa Polistes versicolor para uma área urbana, acompanhando sua aceitação e desenvolvimento neste ambiente. Colônias de $P$. versicolor foram transferidas para abrigos artificiais compostos por potes plásticos fixados a uma haste de madeira. Foi feito um acompanhamento da população através de observação direta, três vezes por semana, durante 14 semanas. Houve $75 \%$ de aceitação para os abrigos artificiais, contudo verificou-se estresse da transferência pela perda de adultos e por comportamento de larvifagia nos primeiros dias após a transferência. A partir da segunda semana, os ninhos tiveram um evidente crescimento populacional até a formação de agregados de inverno quase 4 meses depois. Este é o primeiro estudo que registra a transferência bem sucedida de $P$. versicolor em praças urbanas. Nossos resultados indicam que o método empregado é prático, de baixo custo e com potencial para prover serviços ecológicos e econômicos a longo prazo sem a necessidade de atenção ou manutenção intensa.
\end{abstract}

Palavras-chave: Transferência. Manejo. Aedes aegypti. Controle biológico.

\begin{abstract}
The social wasps are rarely used as biological control agents, partially due to the lack of knowledge on them and their poor reputation, and because it is not known how a species reacts to the transference of its nests. Our study aimed to transfer Polistes versicolor nests to an urban area and to monitor its acceptance to and development in the new environment. $P$. versicolor colonies were transferred to artificial shelters made of plastic containers fixed to a wooden shaft. Population data was monitored by directly observing the nests thrice per week for 14 weeks. There was a $75 \%$ acceptance rate for the artificial shelters, despite the observation of stress-related behaviors such as adult population reduction and larviphagy in the days following the transference. From the second week on, nests showed population growth up to the formation of winter aggregates, almost 4 months post-transference. This is the first report of the well-succeeded transference of $P$.

\footnotetext{
${ }^{1}$ Trabalho premiado no Seminário de Iniciação Científica da UFJF de 2014.

${ }^{2}$ Department of Zoology, University of Otago.340 Great King Street, PO Box 56, Dunedin 9054, New Zealand.

${ }^{3}$ Laboratório de Ecologia Comportamental e Bioacústica, Depto. de Zoologia, ICB, UFJF.

${ }^{4}$ Laboratório de Ecologia Comportamental e Bioacústica, Depto. de Zoologia, ICB, UFJF.
} 
versicolor colonies to shelters in urban parks. Our results indicate that this method is practical, has a low cost and shows potential to provide long term ecological and economical services without the need of constant attention or intensive maintenance.

Keywords: Transference. Management. Aedes aegypti. Biological control.

\section{INTRODUÇÃO}

Os himenópteros sociais (formigas, abelhas e vespas sociais) figuram entre os insetos mais estudados pela ciência. Isto se deve não apenas pelo interesse nos mecanismos evolutivos que levaram ao surgimento da eussocialidade nestes grupos (WILSON, 1971), mas também pela importância ecológica e econômica gerada por sua presença nos ecossistemas que habitam (PREZOTO; CORTES; MELO, 2008; PREZOTO et al., 2016). Espécies de abelhas sociais, por exemplo, são reconhecidas pela importância no processo de polinização (KLEIN et al., 2007) e são exploradas economicamente, já contando com metodologias eficientes bem estabelecidas para seu manejo e criação em ambientes artificiais ou seminaturais (por ex: JANDT et al., 2015).

As vespas sociais, além de um volume de estudos consideravelmente menor se comparado às abelhas, possuem ainda uma percepção geral negativa pela sociedade humana, que tende a relacionar vespas apenas à sua capacidade de ferroar e não ao seu potencial benéfico econômico e ecológico (SUMNER; LAW; CINI, 2018). Além de predadoras de espécies-praga tanto em agroecossistemas quanto em ambientes urbanos (PREZOTO; MACHADO, 1999; PREZOTO; LIMA; MACHADO, 2005; ELISEl et al., 2010), estudos recentes vêm demonstrando seu potencial como polinizadoras (THOMSON, 2018) e dispersoras de sementes (CHEN et al., 2017).

Existe, assim, uma necessidade de se estimular estudos que foquem no manejo, criação e aplicação de vespas sociais como prestadoras de serviços agroecológicos. Ao contrário do que se vê para abelhas sociais como Apismellifera (ver KLEIN et al., 2007) e espécies do gênero Bombus (por ex: JANDT et al., 2012), que apresentam uma boa aceitação à transferência de seus ninhos a abrigos e a condições artificiais, as vespas parecem ser mais sensíveis a esse processo. Salvo algumas exceções do gênero Polistes em países temperados (JANDT et al., 2015) e Vespula (JANDT; TAYLOR; JEANNE, 2010), as vespas sociais parecem preferir abandonar seu ninho após passar pelo trauma de uma transferência, ou interromper o desenvolvimento da colônia caso confinadas a um ambiente artificial. Alguns esforços com espécies neotropicais apresentaram um sucesso considerável, especialmente quando as colônias são transferidas para ambientes de 
cultivo, onde parecem prosperar (PREZOTO; MACHADO, 1999; ELISEI et al., 2010).

Faltam na literatura especializada exemplos bem sucedidos de transferência de ninhos como Polistes ou Mischocyttarus para ambientes altamente antropizados, como praças urbanas. Este ambiente também sofre com a presença de insetos-praga, com destaque para vetores de doenças como no pernilongo Aedes aegypti, vetor da dengue (NATAL, 2002). Uma vez que as vespas são predadoras potenciais de insetos do grupo Diptera (PREZOTO; LIMA; MACHADO, 2005), o manejo de colônias de vespas sociais para áreas estratégicas pode ser uma estratégia de controle de populações de Aedes.

Visando atender a essa necessidade e contribuir com o estabelecimento de uma nova metodologia, nosso estudo visou a transferência de colônias da vespa social Polistes versicolor (Olivier, 1791) para uma área de praça urbana, analisando sua aceitação ao novo ambiente e seu desenvolvimento populacional a longo prazo após a transferência. A partir de nossos resultados, pretendemos prover suporte ao uso desta metodologia para o gênero Polistes no contexto neotropical.

\section{MATERIAL E MÉTODOS}

\section{1 Área e período de estudo}

Este estudo foi realizado no campus da Universidade Federal de Juiz de Fora,

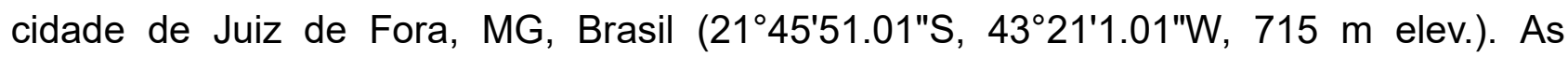
colônias de $P$. versicolor foram localizadas numa residência suburbana no bairro Nova

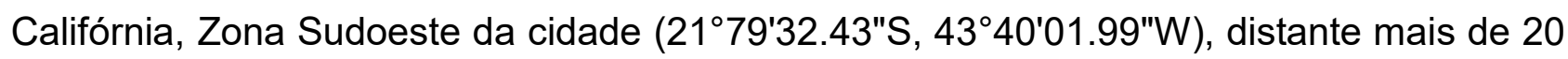
quilômetros do local de transferência. A área para onde as colônias foram transferidas, no campus da UFJF, é uma área de praças urbana; a vegetação próxima é caracterizada como mista com predominância de Pinus elliottii(Engelm.) (CARVALHO et al., 2014).

A coleta das colônias e sua instalação nos abrigos artificiais se deu no dia 19/01/2013. O acompanhamento populacional foi feito por 14 semanas, finalizando-se no dia 05/07/2013. O período de estudo compreendeu a estação Quente e Úmida, uma vez que a área é classificada por Köppen como cwa (ALVARES et al., 2013), com a porção final (Maio-Junho) pertencendo à estação Fria e Seca.

\subsection{Abrigos artificiais}

Os abrigos se constituíram de uma caixa de plástico branco medindo 11 x 15 x 
$10 \mathrm{~cm}$, com as faces inferior e frontal removidas para facilitar a saída e retorno de forrageadoras ao ninho, e a observação das colônias. A parte traseira da caixa foi afixada numa haste de madeira medindo com 1,5 $\mathrm{m}$ de altura, posicionado perpendicularmente ao solo (Figura 1). Os abrigos artificiais foram dispostos linearmente, tês metros entre si.

Figura 1-Abrigos artificiais usados para alojar colônias de Polistes versicolor após sua transferência. A: abrigo artificial. B: ninho de $P$. versicolor em detalhe

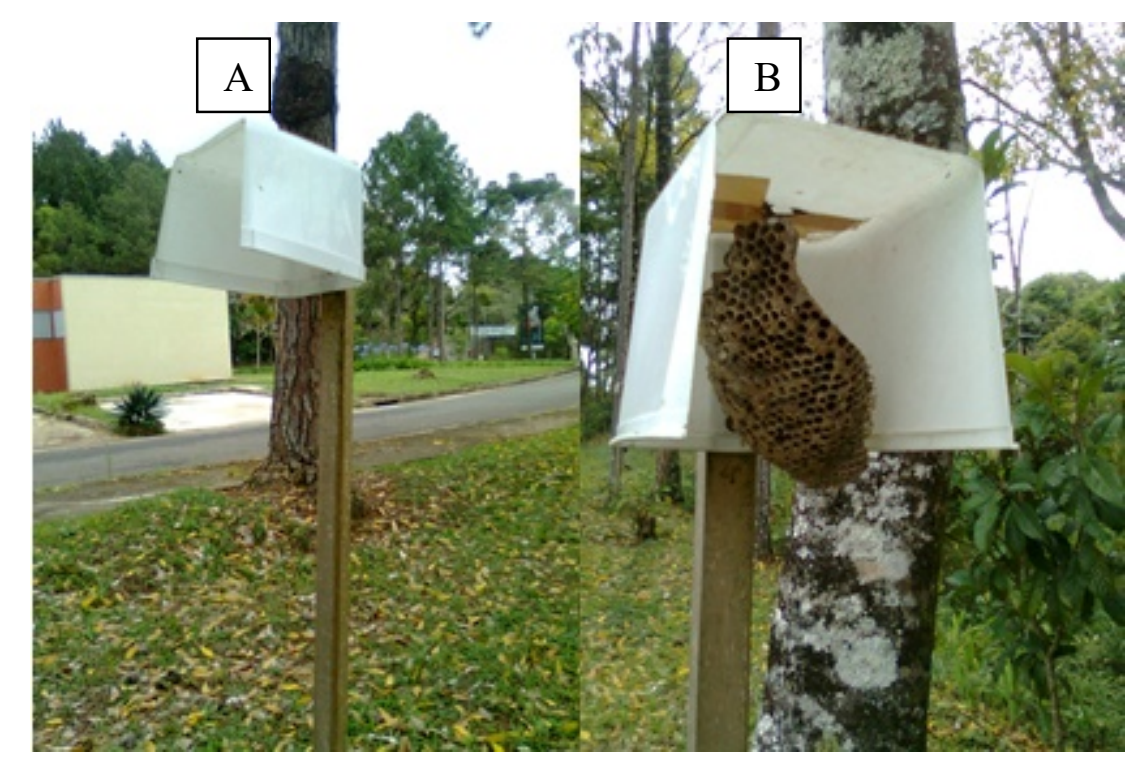

Fonte: elaboração própria.

\subsection{Coleta e transferência de colônias}

O processo de transferência seguiu a metodologia estabelecida por Prezoto e Machado (1999). A coleta dos ninhos se deu 30 minutos após o pôr-do-sol, assegurandose, assim, o término da atividade de forrageio (RICHTER, 2000). Os ninhos foram ensacados com sacos plásticos transparentes, fechados ao redor do pedúnculo que liga a estrutura ao substrato; o pedúnculo era, então, manualmente rompido, preservando sua estrutura da melhor forma possível. Foram coletadas quatro colônias no estágio de pósemergência (CARPENTER; MARQUES, 2001).

As colônias foram mantidas no interior dos sacos plásticos durante o transporte até o local de estudo, onde a fixação das colônias aos seus abrigos artificiais se deu imediatamente. Os ninhos foram separados das vespas adultas dentro dos sacos, permitindo sua retirada e manipulação. Um alfinete foi transpassado na extremidade livre do pedúnculo dos ninhos e, então, usado como apoio para a aplicação de fitas adesivas, fixadas na face interior traseira dos abrigos (Figura 1). Os sacos plásticos com as vespas 
adultas foram, então, ligados aos abrigos por fitas adesivas, limitando a mobilidade das vespas e estimulando sua proximidade com seus respectivos ninhos e imaturos, e aclimatando-as ao novo abrigo e desestimulando o abandono imediato devido ao estresse da manipulação. As colônias permaneceram fechadas até o início da manhã seguinte, quando os sacos foram removidos, completando o processo de transferência.

\subsection{Registro populacional}

Para averiguar a aceitação das colônias aos abrigos artificiais e medir seu desenvolvimento, foi feito um registro do tamanho do ninho (número de células) e das populações (número de adultos) através da observação direta das colônias, três vezes por semana até o fim do estudo. Além disso, a população adulta de cada ninho foi verificada imediatamente antes da transferência para identificar um possível abandono de indivíduos devido ao processo de manipulação. O registro foi sempre realizado logo após o nascer do sol, antes do início da atividade de forrageio das vespas.

\section{RESULTADOS}

Durante a liberação das colônias de $P$. versicolor na manhã do dia 20/01/2013, em todos os abrigos, as vespas adultas permaneceram junto aos seus ninhos, exibindo a postura típica de alarme. A ausência de uma tentativa imediata de dispersão foi considerada um bom sinal de aceitação inicial aos abrigos artificiais. Ao longo do estudo, apenas na Colônia $4(n=1,25 \%)$ abandonou o ninho, dois dias após a transferência. Nas demais colônias $(n=3)$, as populações permaneceram junto aos respectivos ninhos até o fim da observação, resultando numa taxa de aceitação aos abrigos igual a $75 \%$.

Após a transferência, foram registradas evidências de estresse causado pelo processo realocação dos ninhos. Na Colônia 3, houve perda de dois adultos e; na Colônia 2, foram observadas vespas adultas praticando a larvifagia, processo de canibalização das próprias larvas, no primeiro dia após a transferência.

Embora não tenha sido verificado um aumento no numero de células, houve um notável crescimento populacional ao longo do estudo, conforme visto na Figura 2. Nas colônias 2 e 3, houve a formação de agregados de inverno durante o mês de Maio/2013. A Colônia 1 permaneceu ativa até o fim das observações (05/07/2013).

\section{DISCUSSÃO}


Embora a viabilidade de transferência de colônias de Polistes já tenha sido demonstrada (PREZOTO; MACHADO,1999; ELISEI et al., 2010), a literatura existente é focada apenas em abrigos localizados em áreas de cultivo. A reprodução de colônias de vespas sociais em laboratório ainda é virtualmente inexistente, embora exista um esforço de grupos de pesquisa ao redor do mundo para a criação de metodologias (comunicado pessoal dos autores); a obtenção de colônias para manejo, portanto, se dá com a retirada das mesmas de seu habitat natural. Mesmo sendo amplamente distribuídas pelo território brasileiro (BARBOSA et al., 2016), dificilmente vespas como Polistes ocorrerão naturalmente em densidades elevadas o suficiente para que seja possível coletá-las a fim de uma aplicação extensa (o baixo conjunto amostral do presente estudo sendo uma evidência deste problema). Assim sendo, mesmo com eficácia comprovada, dificilmente o uso de vespas sociais será a via principal em um sistema de manejo integrado de pragas agrícolas no cenário científico atual.

Em contrapartida, expandir a presença de colônias de vespas sociais em ambientes heterogêneos e menos extensos, como praças, parques e hortas familiares, é uma alternativa mais viável. Vespas sociais são predadoras generalistas e oportunistas (RICHTER, 2000), capazes de capturar uma ampla diversidade e volumes consideráveis de presas em ambientes urbanos (PREZOTO; LIMA; MACHADO, 2005). Existe um potencial teórico significativo para a aplicação de vespas como Polistes em ambientes antrópicos, seja para o controle de pragas de hortaliças ou para auxiliar no combate a insetos vetores (PREZOTO; LIMA; MACHADO, 2005). Por outro lado, a heterogeneidade dos ambientes urbanos e a possível menor densidade de presas disponíveis quando comparadas aos cultivos pode ser uma barreira para o estabelecimento de colônias em abrigos. Este estudo traz, portanto, no melhor de nosso conhecimento, a primeira evidência publicada da viabilidade de transferir colônias de Polistes para uma área urbana.

Apesar do estresse da manipulação (visto na ocorrência de larvifagia e possivelmente na perda de adultos), as colônias que exibiram tais sinais não apenas se recuperaram dessa perda inicial, como demonstraram expressivo crescimento populacional durante o estudo. Vale notar que para a Colônia 4, única a demonstrar rejeição ao abrigo artificial, foi possível evidenciar o abandono logo durante o primeiro registro populacional; esse resultado é coerente com relatos prévios de que a primeira semana pós-transferência é o período crítico para a aceitação dos abrigos (ELISEl et al., 
2010), e que, passado esse período, o desenvolvimento das colônias remanescentes é uma previsão confiável. Embora nosso pequeno conjunto amostral não permita uma estimação significativa da aceitação dos abrigos artificiais, o índice de $75 \%$ é muito próximo ao encontrado no trabalho de Prezoto e Machado (1999) e representa uma evidência do potencial de sucesso do método em áreas urbanas.

Uma segunda evidência do sucesso da transferência é confirmada pelo crescimento populacional das colônias no período (Figura 2). Apesar da diferença das populações entre as três colônias, pode-se afirmar que isto é um reflexo da variabilidade natural desses aspectos dentre as populações de $P$. versicolor. Além disso, as populações apresentaram um crescimento típico do ciclo de colônias de vespas sociais neotropicais durante a estação quente, com um aumento súbito no número de imaturos

Figura 2 - Média populacional semanal de colônias de Polistes versicolor que aceitaram sua transferência para abrigos artificiais (Colônias 1, 2 e 3) após ao longo do período de estudo (19/Janeiro a 5/Julho de 2013) no campus da Universidade Federal de Juiz de Fora, Juiz de Fora-MG, Brasil. "Dados populacionais de imaturos (ovos, larvas e pupas) não foram coletados pré-transferência; łpopulação adulta formando agregado de inverno

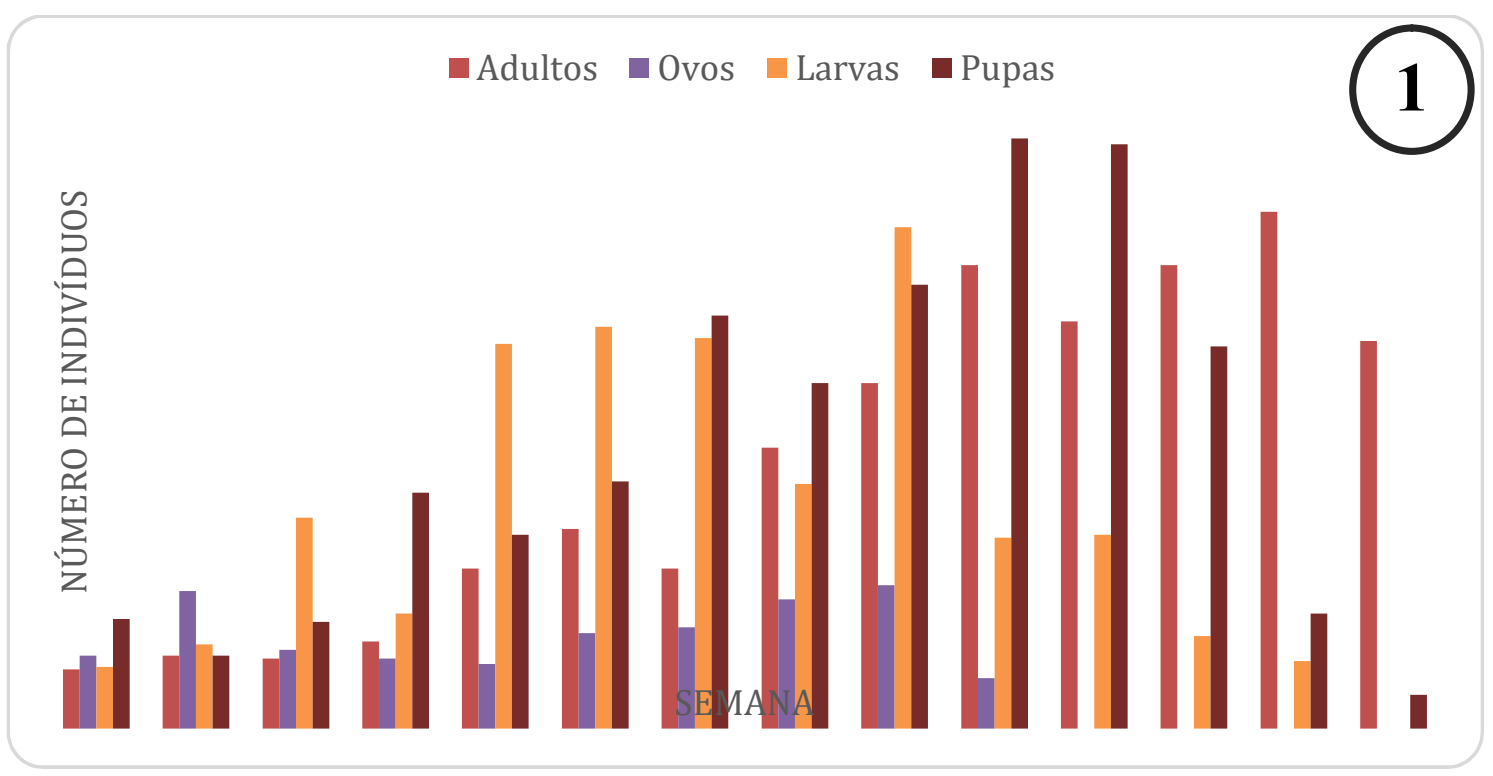



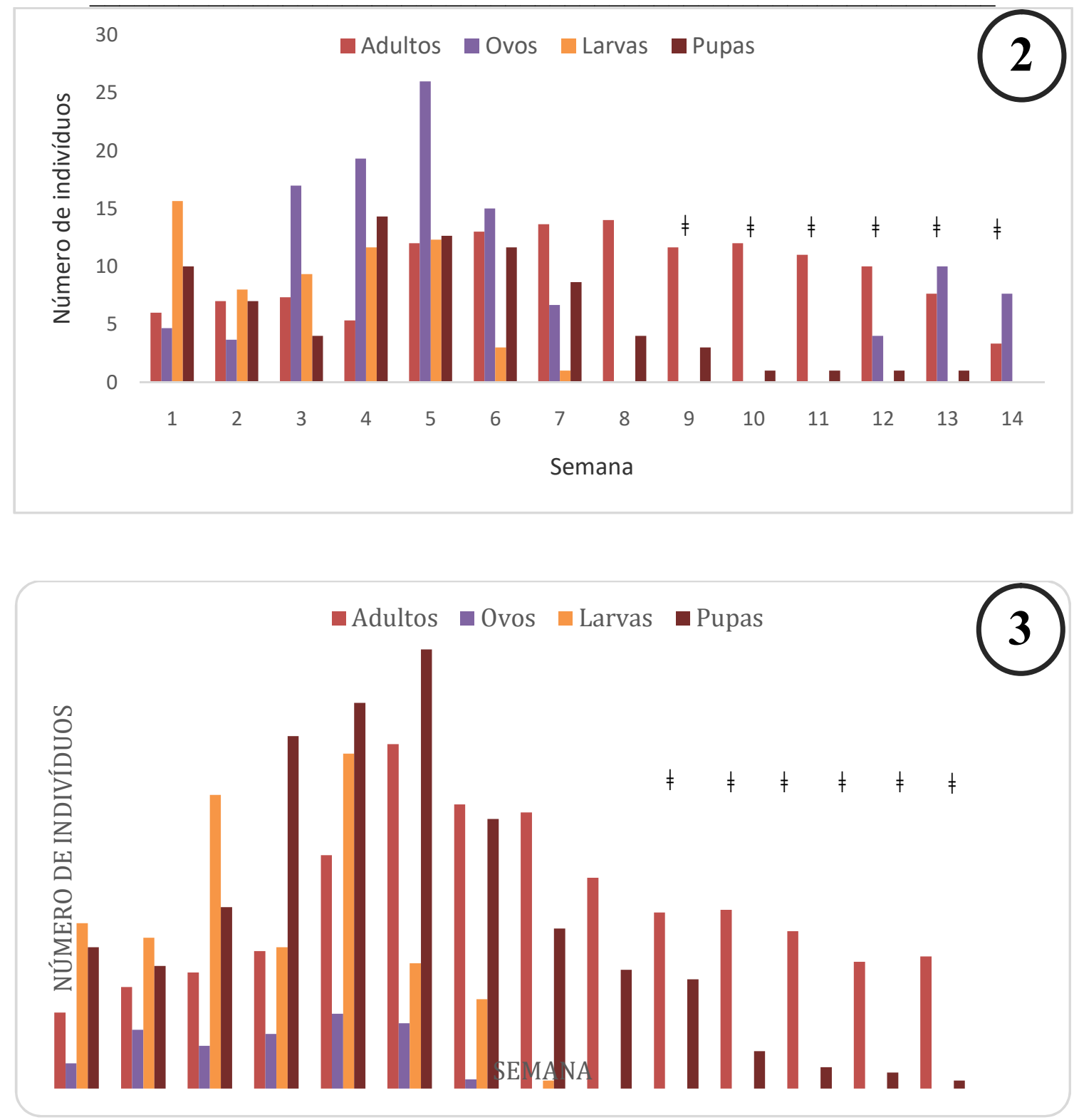

Fonte: Elaboração própria.

nas semanas iniciais e sua diminuição progressiva conforme as colônias se aproximavam da estação fria. Esse comportamento pode ser visto como um sinal de boa adaptação à condição dos abrigos artificiais.

A formação de agregados de inverno nas Colônias 2 e 3 é outro sinal de que as colônias são capazes de manter um ciclo sincrônico típico após sua transferência (GOBBI; ZUCCHI, 1980). Os agregados, condição em que a atividade das colônias se torna mínima e a reprodução cessa (CARPENTER; MARQUES, 2001), também marca o fim da vida útil da colônia num cenário de controle biológico, pois qualquer atividade de forrageio nesse ponto será residual. Isso significa que a maior parte das colônias 
transferidas nesse trabalho manteve atividade por um período de quase quatro meses, sem qualquer tipo de manutenção; ainda mais positivo é o observado para a Colônia 1, que, embora demonstrou declínio populacional ao fim das observações, não interrompeu sua atividade por todo o período. Pensando-se no cenário urbano, esse desenvolvimento acompanha os meses mais quentes e úmidos do ano, período crítico também para o desenvolvimento de insetos vetores de doenças como os pernilongos do gênero Aedes (NATAL, 2002). Essa sincronia entre colônias constitui argumento para o uso de colônias de vespas no auxílio do controle de populações de mosquito caso a predação dos mesmos por aquelas seja melhor evidenciada por estudos práticos (PREZOTO; LIMA; MACHADO, 2005).

Ainda como vantagens deste método, observa-se que os abrigos apresentam um custo e esforço de confecção mínimos, além de se mostrarem duráveis, executando eficientemente a proteção as colônias transferidas (mesmo o ninho da Colônia 4, abandonado no início do estudo, se manteve estruturalmente íntegro até o fim das observações). Além disso, não foi possível observar relações agonística entre colônias, sugerindo que a elevação artificial da densidade populacional não é problemática.

\section{CONCLUSÃO}

Este trabalho apresenta a primeira evidência de transferência bem sucedida de colônias de $P$. versicolor para abrigos artificiais em áreas de praças urbanas na literatura. Nossos resultados trazem argumentos que suportam a utilização de um método prático e de baixo custo, com uma taxa de sucesso considerável e o potencial para prover serviços ecológicos a longo prazo sem a necessidade de atenção ou manutenção intensa.

\section{AGRADECIMENTOS}

Os autores agradecem ao Programa PIBIC-CNPq, pela bolsa concedida ao primeiro autor, durante o desenvolvimento deste estudo.

\section{REFERÊNCIAS}

ALVARES, A. C. et al. Koppen's climate classification map for Brazil.

MetereologischeZeitschrift, Stuttgart, v. 22, n. 6, p. 711-728, Dec. 2013.

BARBOSA, B.C. et al. Studies of social wasp diversity in Brazil: Over 30 years of research, 
advancements and priorities. Sociobiology, [S.I.], v. 63, n. 3, p. 858-880, Sept. 2016.

CARPENTER, J. M.; MARQUES, O.M. Contribuição ao estudo dos vespídeos do Brasil (Insecta, Hymenoptera, Vespoidea). Série Publicações Digitais, Universidade Federal da Bahia, Departamento de Fitotecnia, Mestrado em Ciências Agrárias, Cruz das Almas, v. 2, p. 147, 2001. 1 CD-ROM.

CARVALHO F. A. et al. A comunidade arbórea regenerante de um 'ecossistema emergente' dominado pela espécie invasora Pinus elliottiiEngelm. Interciencia, [S.I.], v. 39 , n. 5, p. 307-312, maio 2014.

CHEN, G. et al. Seed dispersal by hornets: An unusual insect-plant mutualism. Journal of integrative plant biology, [S.I.], v. 59, n. 11, p. 792-796, 11 July 2017.

ELISEI, T.T. et al.Uso da vespa social Polistes versicolor no controle de desfolhadores de eucalipto. Pesquisa agropecuária brasileira, Brasília, v. 45, n. 9, p. 958-964, set. 2010.

GOBBI, N.; ZUCCHI, R. A. OntheecologyofPolistes versicolor (Olivier) in southernBrazil (Hymenoptera, Vespidae, Polistini)l: PhenologicalAccount. Naturalia, São Paulo, v. 5, p. 97-104. 1980.

JANDT, J.M. et al. Individual bumblebees vary in response to disturbance: a test of the defensive reserve hypothesis. InsectesSociaux, France, v. 59, n. 3, p. 313-321, aug. 2012.

JANDT, J.M.; TAYLOR, B.; JEANNE, R.L. Temperature and forager body size affect carbohydrate collection in German yellow jackets, Vespulagermanica (Hymenoptera, Vespidae). InsectesSociaux, France, v. 57, n. 3, p. 275-283, aug. 2010.

JANDT J. M. et al. Lab rearing environment perturbs social traits: a case study with Polistes wasps. Behavioral Ecology, United Kingdom, v. 26, n. 5, p. 1274-1284, 1 sept. 2015

JEANNE, R.L. The adaptiveness of social wasp nest architecture. The Quarterly Review of Biology, Chicago, v. 50, n. 3, p. 267-87, Sept.1975.

KLEIN, A. M. et al. Importance of pollinators in changing landscapes for world crops.

Proceedings of the Royal Society of London B: Biological Sciences, United Kingdom, v. 274, n. 1608, p. 303-313, 7 Feb. 2007.

NATAL, D. Bioecologia do Aedes aegypti. Biológico, São Paulo, v. 64, n. 2, p. 205-207, jul./dez. 2002.

PREZOTO, F. et al. Agroecossistema e o serviço ecológico dos insetos na sustentabilidade. Sustentabilidade: Tópicos da Zona da Mata Mineira. 1. ed. Juiz de Fora: Real Consultoria em Negócios Ltda., p. 19-30, 2016.

PREZOTO, F.; CORTES, S.A.O.; MELO, A.C. Vespas: de vilãs a parceiras. Ciência Hoje, Rio de Janeiro, v. 43, n. 253, p. 70-72, 1 out. 2008. 
PREZOTO, F.; LIMA, M.A.; MACHADO, V.L.L.

SurveyofpreyscapturedandusedbyPolybiaplatycephala (Richards) (Hymenoptera: Vespidae, Epiponini). Neotropical Entomology, Londrina, v. 34, n. 5, p. 489-851, Sept./Oct. 2005.

PREZOTO, F.; MACHADO, V.L.L. Ação de Polistes (Aphanilopterus) simillimusZikán (Hymenoptera, Vespidae) no controle de Spodopterafrugiperda (Smith) (Lepidoptera, Noctuidae). Revista Brasileira de Zoologia, Curitiba, v. 16, n. 3, p. 841-850, 1999.

RICHTER, R. Social Wasps (Hymenoptera: Vespidae) ForagingBehavior. Annual Review of Entomology, Palo Alto, v. 45, p. 121-150, Jan. 2000.

SUMNER, S.; LAW, G.; CINI, A. Why we love bees and hate wasps. Ecological Entomology, v. 43, p. 836-845, 2018.

THOMSON, D.M. Effects of long-term variation in pollinator abundance and diversity on reproduction of a generalist plant. Journal of Ecology, in press. 28 July 2018.

WILSON, E.O. The insect societies. Cambridge: Belknap Press of Harvard University, 1971. 562 p. ISBN 9780674454903. 